\title{
Emerging Technologies and the Accounting Profession: Trends and Topics for Practitioners to Consider
}

\author{
Sean Stein Smith \\ Assistant Professor, Lehman College \\ City University of New York (CUNY)
}

Received: April 8, 2019 Accepted: May 7, $2019 \quad$ Published: June 1, 2019

doi:10.5296/ajfa.v11i1.14627 URL: https://doi.org/10.5296/ajfa.v11i1.14627

\begin{abstract}
Emerging technology and technology tools such as blockchain, artificial intelligence, robotic process automation, and automation at large continue to drive change and disruption throughout the broader financial services landscape. While numerous articles, webcasts, and books have been put together analyzing these different topics and the implications they might have on different areas of the professional landscape, this article proposes a different approach. Instead of focusing on definitions, terminology, or assessing potential impacts at a higher level or through a broad scope of possible applications, this research examines certain specific trends and aspects of these technologies and the impacts they will generate on the profession. Definitions and work terminology will, of course, be a part of this research, but that does not form the core of this piece. Rather, and written with both a practitioner and academic audience in mind, certain emerging topics such and applications built on top of blockchain technology, as well as implementation issues connected to other emerging issues, are the core of this piece.
\end{abstract}

Keywords: Blockchain, cryptocurrencies, accounting, strategic, stablecoins 


\section{Introduction}

Approaching the area of emerging technologies in such a definitive, and somewhat narrow lens may appear to some as artificially narrow or limiting, but that is an incomplete view. Simply put, numerous research, studies, and papers examine the potential of emerging technology to have sweeping changes at different phases of the financial services landscape, and in order to add substantially to this existing literature base, this research examines other area. Be they next step applications such as stablecoins, the assemblage of proposed best practices, or the compilation of questions and considerations to take into account when implementing said technology tools, this research is meant to be further developed, refined, and utilized over time. In other words, the underlying goal of this research and analysis embedded therein is not to simply outline broader guidance or policy, but to analyze specific iterations of advanced technologies and provide specific proposed solutions to address pain points along the way. That said, prior to reviewing these situations and applications, it appears appropriate to first establish working definitions for the purposes of this paper and further discussion. This research and analysis contained within this article were developed and written with both an academic and practitioner audience in mind. Drilling down, and taking into account that the blockchain ecosystem continues to evolve rapidly, the purpose of this research is to provide readers and users with appropriate working definitions and terminology to conduct further analyses and research.

\section{Working Definitions}

Not meant to be all inclusive nor uniquely authoritative in nature, the definitions included below should be used as a framework and starting point for further conversation, analysis, and research in this space.

\subsection{Blockchain}

For the purposes of this research blockchain can be thought of as a decentralized and distributed database for the sharing of information between network members. This information is shared and updated on a nearly continuous basis, and is done so utilizing various forms of encryption. To date, these encryption protocols have proved extremely resistant to hacking attempts, and has generated substantial innovation throughout the business landscape (Lewis, McPartland, and Ranjan, 2017).

\subsection{Cryptocurrencies}

Cryptocurrencies represent perhaps the most well known and commonly used application to run on blockchain technologies, but are not equivalent in nature. In other words, in order to cryptocurrencies to function, they must utilize an underlying blockchain platform. Cryptocurrencies are, in theory, digital assets that can be used as currency alternatives but are not backed or supported by a centralized institution such as a national government or central bank (Sontakke \& Ghaisas, 2017).

\subsection{Artificial intelligence}

Artificial intelligence, or AI, represents a computer program or suite of programs that are able to either augment or replace human oversight and engagement within either an aspect of a 
process or an entire business process.

\subsection{Robotic Process Automation}

Robotic Process, or RPA, can be summarized as the implementation of certain software programs or tools to help with the automation of business processes within an organization. Additionally, it is also perceived or viewed as a stepping stone to a full artificial intelligence implementation program.

\section{Emerging Topics of Consideration}

By any objective measure there has been much written and discussed about the implementation and integration of technology throughout the financial services landscape recently. As this integration continues, as it appears it will virtually without interruption, it is logical to conclude that the issues, opportunities, and obstacles that must be addressed will also change and evolve as well (Borthick \& Pennington, 2017). This section of research breaks down and analyzes both what these specific applications and iteration of technology are, as well as examining some key considerations and questions that need to be addressed by practitioners and additional research moving forward. The first, and possibly most relevant to the profession in terms of reporting, taxation, and compliance for the financial purposes is the growing interest and investment in an asset class called stablecoins. Developed and refined from digitally native cryptocurrencies, these coins and the asset class they represent pose a unique set of questions for practitioners.

\subsection{Stablecoins}

A relatively recent entrant and development in the cryptocurrency landscape, a stablecoin - to summarize concisely - is a cryptocurrency that seeks to either reduce or eliminate altogether the price volatility and uncertainty associated with traditional or first generation cryptocurrencies such as bitcoin. This may seem like a minor change since many investors treat cryptocurrencies as an investment rather than a currency, but in order to facilitate a more mainstream adoption of cryptocurrency assets for both investing and commercial purposes, price volatility must be reduced. Additionally, from strictly an accounting perspective it becomes easier to record, report, and communicate what is occurring with certain types of assets (including stablecoins) if the prices are not fluctuating as wildly as they had in the past (Wieczner, 2018). Interest and investment in this space is clearly indicated by both the launching of the first U.S. based institutional platform to invest in cryptocurrencies by Fidelity in late 2018, and the granting of a banking custodial license to the cryptocurrency exchange Coinbase, also in 2018. Such developments and announcements only represent the proverbial tip of the iceberg in terms of financial and institutional interest in this space, and also reinforces the statement that these are not simply academic concerns, but market driven realities.

That aside, there are a few key considerations and questions that practitioners must be able to ask and address prior to offering advisory services in this fast moving space, and a few of these items are included below

First, what is the underlying stabilizer for this stablecoin, or state a different way, what asset is acting as the volatility reducing agent for the stablecoin in question. Whether the underlying stabilizer is a fiat currency, a physical commodity, or a different stablecoin will have an effect 
on how these items are reported and classified (Meyer, 2018). Drilling down specifically, one item that should be taken into account is how stablecoins are classified versus native cryptocurrencies such as bitcoin or ether. These cryptocurrencies have, based on the white papers and publications that are available for public consumption, tended to be learning toward accounting categorization as intangible assets versus other types of accounting classification. Such consideration and classification may make sense when viewed through how traditional cryptocurrencies have been categorized by regulatory bodies such as the SEC and CFTC, but is an incomplete view of how stablecoins operate and interact with the broader cryptocurrency ecosystem.

If a stablecoin is underpinned by a fiat currency, for example, then it might make sense to treat it and classify it as a currency itself, rather than as an investment or property as is currently the case with cryptocurrencies. This may seem like a minor classification or accounting change in the grand scheme of the financial ecosystem, but can have large ripple effects as to how these stablecoins are used in the financial systems as well as how they are classified, reported, and analyzed by different end users. Increased standardization will also help enable the anti-money laundering aspects of different cryptocurrencies shift from pilot phases to enterprise ready applications (Lai, 2018). Such differentiation is perhaps most clearly demonstrated by the increased institutional interest and investment into the cryptocurrency space, including using stablecoins as collateral for fiat based financial instruments. This next step utilization in the financial space is interesting from an academic sense, but also has implications for how stablecoins should be classified. If, for example, stablecoins are used in lieu or as the underpinning for a fiat based financial instrument, then these items should be treated as currencies, with the liquidity and lack of tax implications associated therein?

A second key consideration, on top of the what the stabilizing or underlying asset itself actually is, is how the stability if achieved, and whether or not the stablecoin itself is pegged directly to the underlying asset, or is merely associated with it in some other manner. If a stablecoin is linked directly to a commodity or fiat current, and has a claim on those underlying assets, then it again seems logical to conclude that appropriate accounting treatment would be equivalent to the treatment of the underlying asset itself. If, however, there is no direct claim or connection the underlying asset, or the stablecoin holders do not have a legal claim to the underlying asset in the case of a business reversal or downturn, classification as a commodity may seem more appropriate and logical. This is not just an academic issue, with nations like Venezuela, complete with the political and economic instability, launching nationally backed coins and tokens; the rights of token holders and coin holders represents a viable economic business issue. Building on this point, and connecting directly to the next topic of analysis and examination is just how stablecoins can be audited, or how should assurance services should be delivered as related to these coins.

Third, and particularly important in the case of stablecoins, which are connected to an underlying asset and done so for a specific purpose to reduce volatility and uncertainty connected to these cryptocurrencies, are the audit and assurance standards that are being built out around the broader cryptocurrency ecosystem. Even if accounting and financial professionals are not expected to be coders and understand the detailed programming languages 
that drive the individual blockchain platforms, practitioners are going be expected to understand the fundamentals of how different tools and platforms work. Dovetailing and building off of this need and expectation of clients to have audit and report ready documentation, this leads to the next emerging topic of interest that is of focus in this research.

\subsection{Audit and Assurance of Cryptoassets}

As of this research there does not appear to be authoritative in nature or issued by an accreditation body such as the FASB or IASB, but there does appear to be an evolving set of standards and information connected to the field of audit, assurance, and reporting around different cryptoassets. The only guidance available in the United States as of this research is the 2014 memo issued by the IRS that classifies all cryptocurrencies as property for tax and reporting purposes. This uncertainty has led to large amount of debate, analysis, and conversation around the future of how these certain assets and items will be reported, but also creates an opportunity for practitioners willing to analyze and examine the issues pertinent to the broader cryptoasset space (Barnes, 2018). Clearly there will be differentiated points of analysis and consideration depending on the specific asset, but there are several points that tend to straddle different cryptoaset classes. These points of analysis are not meant to determine authoritative accounting classification or reporting guidelines, but to rather pose questions related to how certain cryptoassets and items should be treated and reported.

First, is there a third party way to authorize and verify the custody and valuation of these different assets? For traditional and distributed cryptocurrencies the current methodology for obtaining certification of value, via comparison to current market prices, is akin to that of intangible assets, but that is not a perfect solution given the volatility that has traditional been associated with prices of these different asset. Exchange traded products seek to address some of these issues or concerns, but remain primarily in the introductory stages of market implementation (Crea, Shea, Steingarten \& Cohen, 2018). Adding to the difficulty in using an intangible classification to govern the reporting and documentation of cryptocurrencies going forward are the differences in price depending on what exchange is sourced as the basis for price comparison. Even after the trading environment has matured during 2018, price differentials still exist between different exchanges and these different prices do not tend to reconcile over time. A second consideration that is increasingly important as stablecoins become increasingly integrated is how is the custody of different cryptoassets are verified. Particularly with stablecoins, should valuation be determined based on the underlying asset itself, or be a combination of the underlying asset itself plus any market value that has been allocated to the stablecoin running on top of that stabilizer?

A large part of the audit conversation and process revolves around a few key factors: existence, valuation, and confirmation of counter party balances, and it seems that the refinement of blockchain standards may mirror the effect of XBRL on audit and attestation information (Amin, Eshleman \& Feng, 2018). As the cryptoasset environment continues to evolve and proliferate, there are several additional considerations and factors that should be evaluated as a part of the broader assurance dialogue. Examining how these different components of different audit processes will be influenced by the emergence of these technology forces represents an important step toward developing assurance, attest, and other reporting 
frameworks and standards in this space. Not meant to be authoritative nor all inclusive in nature these considerations should rather be thought of as a starting point to continue the dialogue and analysis of how audit, assurance, and attest can be integrated into this emerging field and areas.

Second, is there a method or vector by which the custody of certain cryptoassets can be verified? This mean seem like a rather obvious question or statement but in the face of the anonymity that accompanies many of the most dominant cryptocurrencies this is not an idle question. In fact, the proliferation and rise of so-called privacy coins - whose sole characteristic and trait is the privacy that it imbues the end user with, will only amplify the importance of this question. For clients and institutions that bank with larger and more established institutions such as Coinbase the obtaining of custody information may become simpler as a result of regulatory shifts and developments focused on those more established players. Ironically, and perhaps harkening back to one of the initial tenants and ideals of the cryptocurrency space, decentralized exchanges and platforms are becoming increasingly common as both individuals and institutions seek to truly leverage the decentralized promise of many of these cryptoassets in not only ownership but trading as well. Regardless of the specific structure of the platform or data stream, practitioners need to have access to some sort of information linking ownership of cryptoassets to a public wallet, and a methodology to connect that public wallet - or even public address connected to that wallet - to an identifiable institution or individual.

Third, and perhaps more forward looking than simply focusing on auditing and attesting to the valuation of different cryptoassets is the role that practitioners can play in the development and implementation of control based solutions around smart contracts and other more advanced applications (Raphael, 2017). A smart contract, distilling the concept down to a fundamental level, represents a series of computer codes and language that can automatically execute certain pieces of - or an entire - transaction associated with a business process. This development and application does appear to hold substantial promise for the profession, but also raises several pertinent issues with regards to control, assurance, and attestation. In the case of a smart contract, for example, it is critically important that both the practitioners involved as well as the end users receiving data via the smart contract have confidence in the surrounding processes. For example, a smart contract may indeed automate parts of a process, but what are the controls in place over which institutions or individuals have control over the data as is it input into the system.

\subsection{Differentiation between AI and RPA}

In addition to blockchain implications and applications that have continued to permeate throughout the broader accounting and finance landscape, it also seems important to recognize the fact that other technology trends are having a potentially disruptive impact on the financial services landscape (Cianni \& Steckler, 2017). Automation, artificial intelligence, and robotic process automation are often categorized, labeled, and discussed as if these processes and programs are so similar as to be classified and treated as the same. That said, and linking back to the working definitions established at the beginning of this research, RPA and AI - while related - are not the same on either a technical or implementation basis. Expanding on these initial working definitions, it appears logical to further differentiate and analyze just how these technology tools and processes are indeed distinct and separable. 
Robotic process automation, although a relatively new entry into the marketplace in terms of the terminology and conversation, has existed in various forms for decades. Contrasted versus the emerging fields of artificial intelligence and machine learning, robotic process automation has indeed been adopted by numerous market participants whose track records that are measurable in decades. No matter which specific organization is referenced or included in the analysis or examination of RPA, the underlying fundamentals are similar, but differentiated from the potentially more paradigm shifting artificial intelligence. While not meant to be all inclusive nor exhaustive, the following characteristics form the foundation from further examination by both practitioner and academic researchers.

First, RPA is a technology system and protocol that can be implemented at a range of organizations without having to disrupt or upend current enterprise resource planning systems. This may seem like a technical detail more interesting to a computer science conversation, but it can have substantial effects on the pace with which different technologies and process can actually be adopted and implemented. The ability to install and run RPA based programs in the background means that practitioners and organizations means that organizations can begin to realize the benefits of this increased automation in a nearly instantaneous manner. Said benefits may very well expand beyond accounting and finance processes, and encompass a much wider range of processes than initially anticipated. Drilling down, some of the following processes may be candidates for RPA implementation.

Human resource and onboarding packages may indeed be customized depending on the organization and the individual employee being brought into the organization, but that surface level analysis, although temping, is incomplete in nature. Many of the processes and procedures; employee training, the explanation of benefits, issuance of access codes and other information, and the integration of prior retirement information into the organization structure are surprisingly similar for every employee. Even more basic tasks such as request for documentation and the processing of said documentation are similar, if not identical from employee to employee no matter what role, area of the organization, or geographic location are specified.

Inventory and re-ordering processes and packages are also relatively standardized at all but the smallest of organizations, with a threshold set and established that in turn triggers a reordering of certain goods. While large swaths of inventory management are indeed automated or otherwise augmented by technology much of actual stocking, verification, and reordering of physical good still involves several manual steps and human oversight. Utilizing an RPA based program to assess current levels of inventory, placing orders to replenish the stock of goods at the organization, and keep counterparties aware of what is occurring is a relatively logical place to implement an automation based solution.

Billing and cross referencing the receipt of payments to the appropriate accounts at an organization also seem to be a logical place to increase the automation of processes. Prior to drilling into specific examples or applications connected to the payment process itself, the overarching importance of increasing the efficiency associated with these processes seems a logical place to begin. In a digitally based environment, the idea and concept of float in a traditional sense, has become less important as paper checks and payment processes are less 
common. That said, while external or paper based float may have been reduced in nature, it is interesting that the concept of internal float still appear to be an issue facing many organizations. Not an abstract concept to understand, the idea of internal float as it relates to payments and the applying of payments against outstanding balances can improve the bottom line performance of the organization. At larger organizations, even reducing the amount of time spent manually reconciling payments by a number of hours can result in millions in cost savings and other efficiencies.

Legal matters, such as the review and approval of contracts, also seem to be prime candidates for augmentation or automation via a RPA based program, linking back to the earlier conversation around human resources or benefits stating that many of these contracts and agreements are similar, if not identical in nature. Establishing set processes and protocols for the drafting, review, and resolution of certain contractual matters will - in addition to reducing frustration and the time spent resolving these issues - allow the organization the ability more effectively deploy internal resources. As much promise and potential that automation holds, however, it does appear worthy to point that RPA programming is now the equivalent to artificial intelligence.

Artificial intelligence may initially conjure or bring to mind certain images and ideas that have been put forth by the media and other entertainment venues, but a working definition of AI can be put forward as the following. At the core of the idea and concept, artificial intelligence simply represents a program or suite of programs that can render obsolete or irrelevant the need for human oversight or involvement in a certain process (Sundhararajan, 2018). While automation is certainly prevalent in the marketplace to date, true adoption and implementation of AI by organizations requires several iterative steps that must be completed prior to true AI implementation. First, organizations must have selected the specific type of artificial intelligence that is the most appropriate option for the organization. There are numerous types and classes of artificial intelligence, but there do seem to be three categories that are most applicable to the financial services profession; computational, spatial, and linguistic.

Subsequent to the identification of the most appropriate class and type of artificial intelligence, a series of steps should be completed in order to leverage and unlock the true value proposition of artificial intelligence for an organization. From an accounting and financial professional perspective some of the logical applications of artificial intelligence are connected to fraud detection, continuous reporting, and the identification of patterns from the underlying data (Tysiac, 2017). That said it does seem reasonable to state that, at the current state of development and implementation in the marketplace, many organizations are more well positioned to implement an RPA based program than a true artificial intelligence based solution. As of this research, a primary role and responsibility for financial professionals seems to be closely linked to education and information. Distilling these developments and trends, there are certain frequently asked questions and considerations that should form the basis of a conversation, both between different classes of practitioners as well as between practitioners and clients.

\subsection{Frequently asked Questions and Considerations}


Clearly every organization is different and will necessitate a range of considerations to fully implement and on-board different emerging technologies, but there do appear to several consistent and broad based considerations that should be a part of the accounting and financial services conversation. Viewed from a practitioner perspective this should be used as a framework from which more substantive conversations can be derived. Beginning with blockchain and cryptocurrencies, some of the items that might be discussed are as follows:

1. What type of blockchain solution if applicable to the organization? This presupposes that blockchain is an appropriate technology choice for an organization, but after that initial assessment is complete a next step is to determine what type of blockchain model is the best fit at the current time. Public blockchains, private blockchains, and consortium blockchains have different attributes and characteristics; one of these choices may be a better option than others. An additional consideration that should be taken into account are the relationships with customers, i.e. is it legally possible for the organization to share information between different network members. Also, when implementing blockchain based solutions, an important point to be aware of and take into account is which entities will control the code, especially if external consultants are utilized in the development and implementation.

a. A public blockchain may represent the completely decentralized and distributed model that blockchain supporters most often support, but does not seem to be most appropriate model for enterprise implementation. Whether it is linked to the proof of work model often connected to a public blockchain model, or the association of a public blockchain with cryptocurrencies like bitcoin, organizations might not want to build an enterprise program on this type of blockchain.

b. Private blockchains are more efficient and consume less energy than public blockchains, but are closer to a centralized and traditional database than a true blockchain base solution. Specifically, if other organizations are seeking to leverage some of the benefits of blockchain by sharing and distributing the information, a private blockchain may not be appropriate. In other words, if organizations have to trust the storage and security of information to potential competitors, or even cooperating firms, this raises additional issues of governance and data security.

c. Consortium blockchains are, in essence, a hybrid type of blockchain platform that might seem to be best applicable for broader enterprise adoption as of this research. An analogy that can be used for educational or explanatory purposes is that a consortium blockchain model is a platform that can be used by a number organizations to share data and information with joint ownership of the underlying processes. Although the joint custody of information generates efficiency benefits, it also raises considerations that have to analyzed as they pertain cybersecurity and governance.

2. Cybersecurity and governance issues are also key items that should be considered when implementing either blockchain or automation (be it AI or RPA) based solution or process within an organization. Cybersecurity policies and practices based on simply possessing up to date anti-virus software or enforcing password protocols are insufficient given the rapid pace of change in the business environment at large. Practitioners across different industry lines will 
have to both keep pace with the underlying technology itself as well as the implications of these technologies on current processes.

a. A substantial consideration and factor that should be taken into account, especially as emerging technologies are onboarded and implemented throughout the marketplace, is whether or not the controls in place at the organization are sufficient enough to deal with the acceleration of data processing and reporting. Particularly as new technologies are developed and implemented it is entirely possible for existing controls to either end up bypassed or otherwise ignored as a result of these implementations. Maintaining the integrity and strength of these different controls and platforms is both a core responsibility of accounting professionals as well as a fiduciary responsibility for all professionals.

3. Once the blockchain platform has been decided upon, what are the processes and policies in place to ensure the organizations and individuals are both well qualified to work on the various stages of blockchain projects. If, for example, external consultants were employed to help develop and engineer a new product or service, there are well established policies and processes in place to help safeguard the information covered and discussed between internal and external consultants. Blockchain is, at the core of the idea, simply a computer system and program and the controls in place must be able to control both who has control over the underlying code itself, and which individuals maintain control over the information once it has been implemented.

a. A conversation surrounding the maintenance and ongoing controls linked to both blockchain itself, and the APIs that migrate data stored on the blockchain to existing technology systems must be weaved into the broader control process itself.

b. Are the individuals and firms brought in to consult with the development of the blockchain itself properly insured and covered for potential errors of omissions that do occur.

c. This broader debate surrounding the access and levels of insurance linked both the client organization and external consultants brought in to assist with specific projects are carries through to a more comprehensive conversation around the internal controls over these emerging technologies.

4. Internal control considerations as they are connected to emerging technology issues would seem to pose an apparent contradiction for practitioners (Drew, 2018). On one hand the increased speed and security with which transactions can be processed and stored appears to decrease the need for manual controls or reviews of information. The efficiency gains and benefits associated with this increased speed of transactional processing are relatively clear in nature, but also raise the possibility of internal controls being subverted or overwritten via the implementation of new technology.

a. Specifically, the implementation and adoption of new technologies that help to accelerate the data processing and reporting tools could actually amplify underlying issues and errors that otherwise might have been addressed via manual controls.

b. Taking this into account, and realizing that every organization is different, and that different processes are going to be governed by a variety of forces, this also seems a logical space to raise the issue and importance of documentation. Properly documenting and testing 
the documentation of processes to ensure that current processes are indeed appropriate is an important first step in the developing. Building on this initial documentation, however, it also seems responsibility to develop frameworks and tests to maintain the integrity of controls as technology becomes more integrated.

5. Is it possible to integrate and map the data and information stored on a blockchain platform with current information technology systems? Even with existing technology systems there are often issues with the mapping, transmission, and exporting of information between different modules of the same system. Since the majority of blockchain platforms are based on technology that, although robust in its own right, is still immature and an emerging technology platform, it is important for organizations to identify the appropriate controls and policies to safeguard information. Several considerations that appear to still be open times include, but are not limited to the following:

a. Is the data stored on a blockchain that is private and hosted by an organizing firm, does that mean that the other blockchain members will have to request access to that information?

b. Rather, if the group of organizations have constructed a consortium blockchain model, are the controls and access restrictions equivalent between these different organizations. Since consortium or sector specific models and platforms seem be more and more popular among different industry groups this is not an abstract issue or concern.

\section{Additional Considerations}

Based on the analysis included both in this research and other research projects it should be clear that the accounting and broader financial services landscape continues to change and evolve. Including or producing an all inclusive listing of potential future service offerings is beyond the scope of this, or any other single research piece. That said, there do appear to be several roles and services that financial services are uniquely well positioned to fill as the technological integration and intersection of accounting and financial services continues to increase. Maintaining a focus on the applications of blockchain and automation tools including both artificial intelligence and robotic process automation - the following appear to represent roles and duties financial practitioners will be well versed to fill and occupy moving forward (Jun \& Vasarhelyi, 2017).

First, even as technology becomes increasingly integrated into different roles and organizations, the core responsibilities and competencies of accounting professionals will not change entirely, but will evolve to keep pace with changing expectations. One logical extension and development as a result of these changes is the continued importance of internal controls, especially those pertaining to data governance and other processes. Building controls, maintaining controls, and explaining different and new controls to members of the organizations will continue to remain important for practitioners. This might seem contradictory as technology assumes more of the computational components of different processes, but controls and maintaining adequate controls are actually more important as automation becomes more important. Reinforcing and developing expertise in control development, testing, and monitoring processes represents a potential new service line and revenue opportunity. 
Second, the concept of an audit and other attestation engagements will invariably change and evolve over time as the technology tools and platforms utilized by both organizations and accounting professionals continue to change and become more sophisticated (Alarcon \& $\mathrm{Ng}$, 2018). Continuous auditing and attestation are not as abstract or radical an idea as many initially appear; organizations are already experimenting with such tools to improve the quality and transparency of information. Expanding this perspective, and taking into account the increased interest in sustainability and other more comprehensive forms of reporting, it seems logical to conclude that this more continuous type of reporting will become more mainstream. Especially as organizations, management teams employed therein, and the stakeholders of said firms become increasingly accustomed to continuous reporting, financial professionals will have to keep pace.

Third, the role that practitioners will fill moving forward will be influenced by dual forces that are, in essence, of equal of importance in both United States and international markets. Put simply, clients and other internal partners and stakeholders are going to have to balance the expectations - including hype and excitement associated with emerging technologies - with reality of where technologies actually intersect with current needs. Clearly every organization is different and will have unique needs associated with different emerging technologies, but practitioners will need to posses the following traits. In addition to the technical expertise necessary to understand the tools themselves, the ability to communicate and articulate the implications of these tools will also become more important as clients have needs and expectations. While the underlying aspects and considerations for practitioners and organizations will vary depending on the individual firm, the increasing integration of technology also will develop considerations that are not yet identifiable at this point. Analyzing these potential trends and directions for both accounting practitioners and management teams is something connected to this research as well as future directions.

\section{Future Considerations \& Directions}

This research, like any research connected to an emerging technology such as those documented and analyzed within this paper, is an attempted analysis of emerging technologies and tactics, but should rather form the basis for additional considerations and research. Roles and responsibilities connected to financial practitioners will evolve and change as technology continues to become increasingly integrated within the broader marketplace. Whether the specific technology in question is connected to blockchain options, cryptocurrencies, stablecoins, or automaton trends in general, the overarching trends are similar. First, practitioners employed both within public practice and private industry will be involved in fewer compliance and manual tasks. Such tasks and processes do not generally add value either to the practitioners involved nor the client organization so this automation and tasks elimination appears to be a positive trend. Second, and across the financial services spectrum, the need and demand for practitioners to act in an advisory manner is becoming increasingly clear. Whether it is connected to explaining and analyzing emerging technology trends themselves, or simply serving as a level setting reality check against unrealistic expectations, the shift toward strategic advisor appears to be well underway.

Finally, the need and responsibility for practitioners to engage in continuous learning and 
education does not appear represent a fad, but rather a fundamental change in how professional interact with the broader business environment. Continuing education requirements are already a component of the professional environment, but the pace with which various technologies are changing points to a necessary shift. On top of changing the manner with which practitioners must educate themselves, the need for continuous learning and education also opens a new line of advisory services for forward looking practitioners. On balance, it seems that from the available literature that while the profession itself is in the midst of a change with regards to some core roles and responsibilities, that said changes both eliminate some current roles while simultaneously creating new roles and potential opportunities.

At the core of this research was the desire to be applicable for both practitioners and academics, with concepts as well as market based and derived applications of these new tools. Moving forward, the need for dialogue, analysis, and further examination of both these technology trends and associated implications is relatively clear. The reality that technology is becoming more integrated into financial services is not, in and of itself, aground breaking concept of idea. Rather, and by focusing on a few specific applications and trends related to emerging technology topics, accounting practitioners and researchers can apply and expand on this analysis and findings therein moving forward. Written with both a research and practitioner audience in mind, this research forms the foundation for discussion, experimentation, and further refinement both currently and in the future.

\section{References}

Alarcon, J. L. "John," \& Ng, C. (2018). Blockchain and the future of accounting. Pennsylvania CPA Journal, 3-7.

Amin, K., Eshleman, J. D., \& Feng, C. (Qian). (2018). The effect of the SEC's XBRL mandate on audit report lags. Accounting Horizons, 32(1), 1-27. https://doi.org/10.2308/acch-51823

Barnes, N. D. (2018). Blockchain redux - cracking the code on Cryptocurrencies. Information Management Journal, 52(6), 26-30.

Borthick, A. F., \& Pennington, R. R. (2017). When data become ubiquitous, what becomes of accounting and assurance? Journal of Information Systems, 31(3), 1-4. https://doi.org/10.2308/isys-10554

Carlozo, L. (2017). Understanding blockchain. Journal of Accountancy, 224(2), 1.

Cianni, M., \& Steckler, S. (2017). Transforming organizations to a digital world. People \& Strategy, 40(2), 14-19.

Crea, R., Shea, P., Steingarten, D., \& Cohen, D. (2018). Cryptocurrency exchange traded products: If, when, and how. (cover story). Investment Lawyer, 25(12), 1-15.

Drew, J. (2018). Merging accounting with "big data" science. Journal of Accountancy, 226(1), 47-52.

Jun Dai, \& Vasarhelyi, M. A. (2017). Toward blockchain-based accounting and assurance. Journal of Information Systems, 31(3), 5-21. https://doi.org/10.2308/isys-51804

Lai, K. (2018). Singapore banks using DLT to tackle money laundering. International Financial Law Review, 1. 
Lewis, R., McPartland, J. W., \& Ranjan, R. (2017). Blockchain and financial market innovation. Economic Perspectives, 41(7), 1-17.

Meyer, D. (2018). Bitcoin price jumps as cryptocurrency traders become wary of tether "Stablecoin." Fortune.Com, N.PAG.

Raphael, J. (2017). Rethinking the audit. Journal of Accountancy, 223(4), 29-32.

Sontakke, K. A., \& Ghaisas, A. (2017). Cryptocurrencies: A developing asset class. International Journal of Business Insights \& Transformation, 10(2), 10-17. https://doi.org/10.1080/1540496X.2016.1193002

Sundhararajan, M., Gao, X.-Z., \& Vahdat Nejad, H. (2018). Artificial intelligent techniques and its applications. Journal of Intelligent \& Fuzzy Systems, 34(2), 755-760. https://doi.org/10.3233/JIFS-169369

Tysiac, K. (2017). How to enable audit innovation. Journal of Accountancy, 223(4), 33-35 Wieczner, J. (2018). PwC is backing a USD stablecoin cryptocurrency in challenge to tether. Fortune.Com. 